\title{
Platelet indices as prognostic markers in the critically ill patient
}

\author{
Anusha J. Yadav, Shaikh Mohammed Aslam \\ Department of General Medicine, M S Ramaiah Medical College, Rajiv Gandhi University of Health Sciences, Bangalore, \\ Karnataka, India
}

\begin{abstract}
Platelet indices such as mean platelet volume (MPV), platelet (PLT) count, plateletcrit (PCT), and platelet distribution width (PDW) describe platelet morphology and proliferation kinetics. Our study aimed to evaluate the utility of platelet indices as prognostic markers in critically ill patients. This was an observational, descriptive study conducted on 106 critically ill adults for 24 months in the Medical Intensive Care Units of a tertiary care hospital. Analysis of the data was done using statistical software R version 3.6.0 and MS Excel. The mean age of patients was 42.3 \pm 5.8 years. Non-survivors had lower PLT count and PCT when compared to survivors. PDW and MPV were higher for non-survivors when compared to survivors. PLT count of $<90,000$ cells/cu mm displayed the highest sensitivity (94\%), while PDW demonstrated the highest specificity (96\%) in predicting mortality among critically ill patients. Abnormally low PLT count, high PDW, and high MPV values are associated with severe illness and put the patients at high risk of death as compared to patients with normal PLT indices.
\end{abstract}

Correspondence: Shaikh Mohammed Aslam, Department of General Medicine, M S Ramaiah Medical College, Rajiv Gandhi University of Health Sciences, Mathikere, Bangalore 560054, Karnataka, India.

Tel.: +91.9448450678.

E-mail: drmdaslam@yahoo.com

Key words: Platelet count; blood platelets; critical care; mean platelet volume.

Contributions: AJY, literature search, data acquisition and statistical analysis; manuscript editing and review; SMA, definition of intellectual content; AJY and SMA, study concepts and design, clinical and experimental studies, manuscript preparation, guarantors.

Conflict of interests: the authors declare no potential conflict of interests.

Availability of data and materials: data and materials are kept by the corresponding author and are available for consultation upon request.

Ethics approval and consent to participate: the present study was initiated following the grant of approval by the ethical review board of the Institutional Ethics Committee on $18 / 10 / 2016$. Written informed consent was obtained from the patients for their anonymized information to be published in this article. The study protocol adopted conforms to the 1975 Declaration of Helsinki's ethical guidelines as reflected in the prior approval of the institutional ethics committee.

Received for publication: 14 October 2020.

Revision received: 10 December 2020.

Accepted for publication: 13 December 2020.

This work is licensed under a Creative Commons Attribution NonCommercial 4.0 License (CC BY-NC 4.0).

${ }^{\circ}$ Copyright: the Author(s), 2021

Licensee PAGEPress, Italy

Italian Journal of Medicine 2021; 15:34-40

doi:10.4081/itjm.2020.1388

\section{Introduction}

Platelets are an important constituent of blood, playing a significant role in physiological and pathological processes such as coagulation, thrombosis, inflammation, and maintenance ofvascular endothelial cell integrity. ${ }^{1}$ In addition to their important role in hemostasis and thrombosis, accumulating evidence demonstrates that platelets contribute to the inflammatory process, microbial host defense, wound healing, angiogenesis, and remodeling. ${ }^{2}$ Platelet indices such as platelet count (PLT), mean platelet volume (MPV), platelet distribution width (PDW), and plateletcrit (PCT) are simple indices that any 3-part differential cell counter can calculate. However, assessment of immature platelet fraction can be done only at advanced centers using more complex cell counters. ${ }^{3}$ These are commonly used to measure the total number of platelets, their morphology and proliferation kinetics, and can be applied in the diagnosis of diseases affecting the hematological system. ${ }^{2}$

A reduction in PLT count is an independent risk factor for critically ill patients in the Intensive Care Unit (ICU). ${ }^{4}$ In addition, thrombocytopenia is included as an independent risk for mortality in the Acute Physiology and Chronic Health Evaluation II (APACHE II) system. ${ }^{4}$

Numerous blood count parameters such as packed cell volume, PCT count, and total leucocyte count are used in various scoring systems for intensive care patients, such as multiple organ dysfunction scores, sequential organ failure assessment scores, and logistic organ dysfunction scores..$^{5}$ Lately, numerous studies ${ }^{2,5-}$ ${ }^{7}$ have suggested an association between these indices and platelet activation, which is an independent risk fac- 
tor in the critically ill patient. Increased platelet activity can be diagnosed by a raised platelet volume, which, in turn, suggests an increased prothrombotic state associated with adverse outcomes in ICU patients. Factors such as thromboxane $\mathrm{A} 2$ have prothrombotic properties and are produced during hemostasis by activated platelets. It increases platelet aggregation as well as stimulates activation of new platelets. Large platelets have increased platelet activity as they produce large amounts of thromboxane A2.

As platelet indices are inexpensive and relatively less time consuming, ${ }^{6}$ they can be ideal for use as prognostic factors for critically ill patients in a country like India, where healthcare resources are scarce. The present research was intended to study platelet indices as prognostic factors in critically ill patients at a tertiary care hospital.

\section{Materials and Methods}

This was an observational, descriptive study conducted for 24 months in the medical intensive care units (MICUs) of a tertiary care hospital on 106 patients. All critically ill adult patients admitted to MICUs were included in the study. Written informed consent was obtained from the patients. Pregnant women, patients with active hemorrhage, hematological diseases (including anemia, hypersplenism, lymphoma or leukemia, and bone marrow disorders), infectious diseases that primarily affect the platelets (dengue fever, malaria, viral fever, etc.), patients who have received radiotherapy or chemotherapy or bone marrow transplantation one month before admission, patients who have used antiplatelet drugs (aspirin, clopidogrel) or other drugs which could reduce platelet count (e.g., non-steroidal anti-inflammatory drugs, etc.) prior to admission were excluded from the study. The purpose of the study was explained to the patients and their representatives, and informed consent was obtained. Thereafter, the patients were assessed. Blood samples for complete blood count, including platelet count, MPV, PDW, and PCT, were sent on admission. Other tests such as arterial blood gas, liver and renal function tests were done. As a part of automated complete blood count, platelet indices were measured. Demographic data, history, examination findings, and relevant laboratory tests were also performed.

Data were analyzed using statistical software R version 3.6.0 and Excel. Continuous variables were represented by mean \pm standard deviation (SD) form. Frequency tables and percentages represented categorical variables. Independent t-test, Mann Whitney U-test, and Kolmogorov-Smirnov test were used whenever the data followed non-normative distribution. Categorical variables were compared using the Chi-square test and odds ratios.

\section{Results}

We conducted the present study on 106 patients for 24 months at a tertiary care hospital.

The mean age of subjects involved in our study was $46.85 \pm 14.08$ (mean \pm SD) years. Figure 1 shows that most of the patients in our study were under the

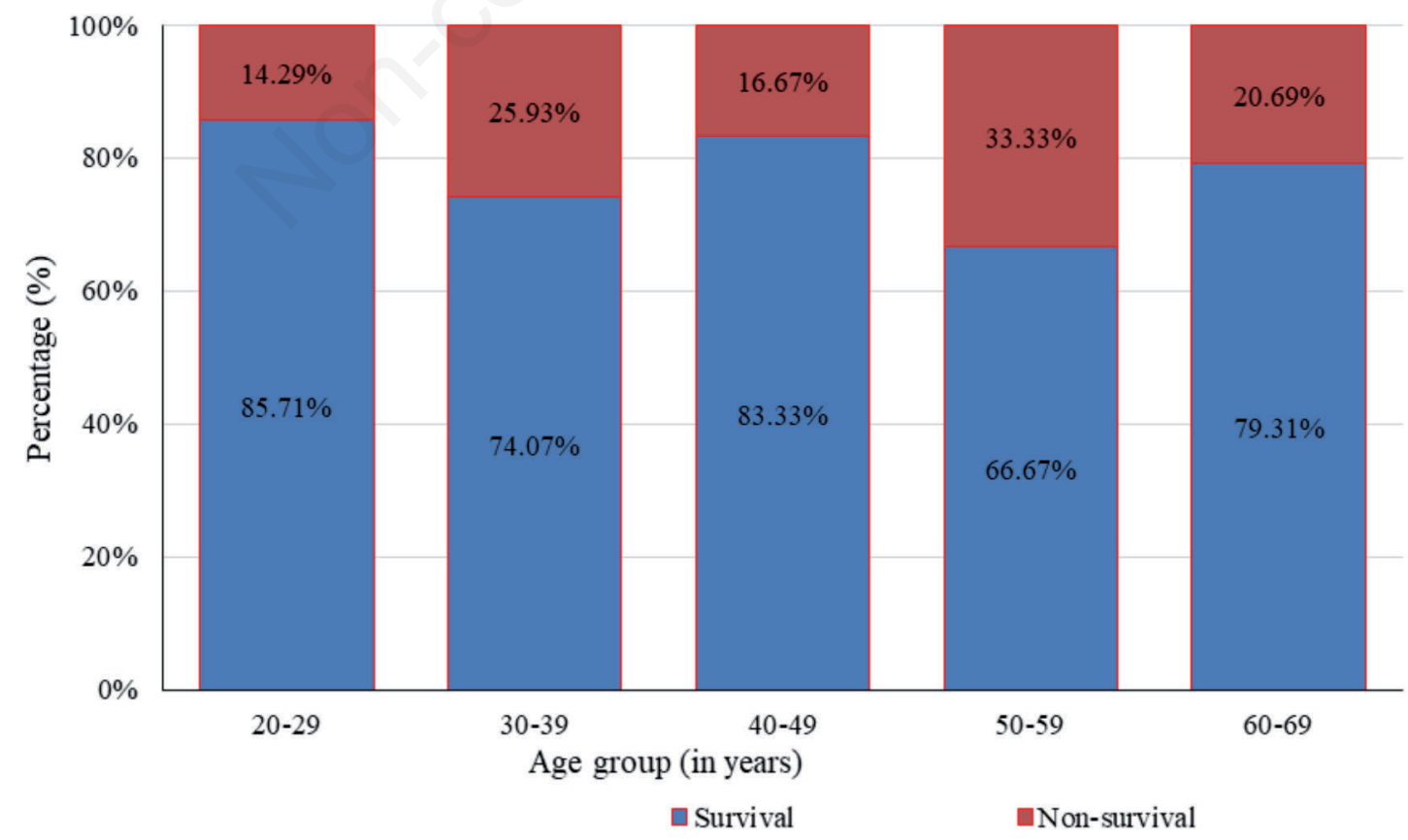

Figure 1. Distribution of study subjects based on age. $\mathrm{X}$-axis: age group (in years); Y-axis: percentage of study subjects. 
age group 40-69 years. Among the total study participants, 54 (50.94\%) were females, and 52 (49.05\%) were males.

Among clinical symptoms studied (Table 1), fever was the most common presenting symptom and was present in $61(57.33 \%)$ patients. Cough was the second most common presenting symptom and was present in $60(56.60 \%)$ patients. Other presenting symptoms included dyspnea $33(31.1 \%)$, vomiting 16 (15.09\%), and abdominal pain $10(9.43 \%)$.

When the patients were studied according to their diagnosis (Table 1), we found out that the most common diseases among people admitted to the ICU were as follows; 36 (33.9\%) patients had pneumonia , 26 (24.5\%) had sepsis , and $23(21.6 \%)$ had an acute exacerbation of reactive airway disease . Among the rest, $12(11.3 \%)$ patients had urosepsis, $6(5.6 \%)$ patients had acute pancreatitis, and $3(2.8 \%)$ patients had viral encephalitis. We observed that the survival rate was higher among the subjects diagnosed with pneumonia or acute exacerbation of reactive airway disease, whereas the survival rate was low for subjects diagnosed with acute pancreatitis or viral encephalitis. Broadly similar survival rates of 76.92 and $77.78 \%$ were noticed among males and females, respectively.

From Table 2, we may conclude that the median of ICU stay was not significantly different between survivors and non-survivors $(\mathrm{P}=0.552)$. The distribution of total leucocyte count $(\mathrm{P}=0.0003)$ and serum creatinine $(\mathrm{P}<0.0001)$ was found to be statistically different between survivors and non-survivors. Also, various platelet indices studied, such as PLT count $(\mathrm{P}<0.000)$, PDW $(\mathrm{P}<0.0002), \mathrm{MPV}(\mathrm{P}=0.0041)$, and plateletcrit $(\mathrm{P}<0.0001)$, were also statistically significant between survivors and non-survivors.

The mean PLT count was lower among non-survivors than among survivors (77,500 $\pm 10,570$ and $132,800 \pm 17,500$ cells/cumm respectively) (shown in

Table 1. Distribution of patients based on clinical symptoms, comorbid conditions, and diagnosis.

\begin{tabular}{|c|c|c|c|c|c|}
\hline Parameter studied & & $\begin{array}{l}\text { Survivors } \\
n=82(\%)\end{array}$ & $\begin{array}{c}\text { Non-survivors } \\
n=24(\%)\end{array}$ & $\begin{array}{c}\text { Total } \\
\mathrm{n}=106(\%)\end{array}$ & P-value \\
\hline Clinical symptoms & $\begin{array}{l}\text { Fever } \\
\text { Cough } \\
\text { Dyspnea } \\
\text { Vomiting } \\
\text { Abdominal pain }\end{array}$ & $\begin{array}{l}46(56.10 \%) \\
48(58.54 \%) \\
22(26.83 \%) \\
09(10.98 \%) \\
06(7.32 \%)\end{array}$ & $\begin{array}{c}15(62.50 \%) \\
12(14.63 \%) \\
11(13.41 \%) \\
07(29.17 \%) \\
04(4.88 \%)\end{array}$ & $\begin{array}{l}61(57.55 \%) \\
60(56.60 \%) \\
33(31.13 \%) \\
16(15.09 \%) \\
10(9.4 \%)\end{array}$ & $0.04 *$ \\
\hline Diagnosis & $\begin{array}{l}\text { Pneumonia } \\
\text { Sepsis } \\
\text { Acute exacerbation of reactive airway disease } \\
\text { Urosepsis } \\
\text { Acute pancreatitis } \\
\text { Viral encephalitis }\end{array}$ & $\begin{array}{l}20(76.92 \%) \\
29(80.56 \%) \\
18(78.26 \%) \\
9(75 \%) \\
4(66.67 \%) \\
2(66.67 \%)\end{array}$ & $\begin{array}{l}6(23.08 \%) \\
7(19.44 \%) \\
5(21.74 \%) \\
3(25 \%) \\
2(33.33 \%) \\
1(33.33 \%)\end{array}$ & $\begin{array}{c}26(24.53 \%) \\
36(33.96 \%) \\
23(21.7 \%) \\
12(11.32 \%) \\
6(5.66 \%) \\
3(2.83 \%)\end{array}$ & $0.001 *$ \\
\hline
\end{tabular}

*Significant at $\mathrm{P}<0.05$ threshold.

Table 2. Comparison between duration of stay and laboratory parameters among survivors and non-survivors.

\begin{tabular}{|c|c|c|c|}
\hline Parameters studied & $\begin{array}{l}\text { Survivors } \\
\quad(n=82)\end{array}$ & $\begin{array}{c}\text { Non-survivors } \\
(n=24)\end{array}$ & P-value \\
\hline Duration of ICU Stay (mean \pm SD) & $7.48 \pm 3.04$ & $8 \pm 3.30$ & 0.552 \\
\hline \multicolumn{4}{|l|}{ Laboratory parameters } \\
\hline Hemoglobin (gm/dL) & $12.38 \pm .93$ & $12.58 \pm 2.00$ & 0.6503 \\
\hline TLC (cells/cu mm) & $13.30 \pm 5.04$ & $16.76 \pm 7.67$ & $0.0003 *$ \\
\hline ESR (mm/1 ${ }^{\text {st }}$ hour) & $60.74 \pm 20.49$ & $55.17 \pm 18.57$ & 0.5208 \\
\hline Serum creatinine $(\mathrm{mg} / \mathrm{dL})$ & $1.88 \pm 0.98$ & $6 \pm 2.00$ & $<0.0001^{*}$ \\
\hline Total bilirubin (mg/dL) & $0.91 \pm 0.17$ & $0.91 \pm 0.18$ & 0.9038 \\
\hline Albumin $(\mathrm{gm} / \mathrm{dL})$ & $3.55 \pm 0.54$ & $3.57 \pm 0.50$ & 0.9728 \\
\hline $\operatorname{AST}(\mathrm{U} / \mathrm{L})$ & $33.62 \pm 6.88$ & $31.46 \pm 6.46$ & 0.2579 \\
\hline $\operatorname{ALT}(\mathrm{U} / \mathrm{L})$ & $33.83 \pm 6.78$ & $34.96 \pm 7.45$ & 0.6942 \\
\hline \multicolumn{4}{|l|}{ Platelet indices } \\
\hline Platelet count (cells/cumm) & $2.02 \pm 0.83$ & $1.15 \pm 0.49$ & $<0.000 *$ \\
\hline PDW (\%) & $13.44 \pm 2.32$ & $15.71 \pm 2.42$ & $<0.0002 *$ \\
\hline MPV (\%) & $12.65 \pm 1.91$ & $14.00 \pm 1.64$ & $0.0041 *$ \\
\hline Plateletcrit (\%) & $0.19 \pm 0.05$ & $0.13 \pm 0.06$ & $<0.0001^{*}$ \\
\hline
\end{tabular}

ICU, Intensive Care Unit; SD, standard deviation; TLC, total leucocyte count; ESR, erythrocyte sedimentation rate; AST, aspartate aminotransferase; ALT, alanine aminotransferase; PDW, platelet distribution width; MPV, mean platelet volume. *Significant at $\mathrm{P}<0.05$ threshold. 
Table 3). The difference was statistically significant with $\mathrm{P}<0.001$. Mean PDW was higher in non-survivors compared with survivors (13.6 \pm 1.63 and $11.24 \pm 2.61$ respectively), with a statistically significant difference $(\mathrm{P}<0.038)$. Mean MPV was higher in non-survivors than in survivors (15.2 \pm 1.46 and $11.46 \pm 2.71$ respectively). The difference was again statistically significant with $\mathrm{P}<0.024$. Mean PCT was lower in non-survivors when compared to survivors $(0.11 \pm 0.031$ and $0.17 \pm 0.048$ respectively), with the difference being statistically significant $(\mathrm{P}<0.017)$.

Among 106 patients studied, 82 (77.36\%) patients survived. Hence the mortality rate in our study was $22.64 \%$.

In Figure 2, the area under the curve for the PLT count was $83 \%$, so PLT count turned out to be a good and accurate measure in predicting the survival status. The area under the curve for PDW was $77 \%$, PDW was also an accurate factor in predicting the survival status. The area under the curve for MPV was $71 \%$, and MPV was also accurate in predicting the survival status. The area under the curve for PCT was $84 \%$, making PCT the most accurate indicator in predicting the survival status.

The survival status was significantly associated with PDW category and MPV category. For patients with PDW $<13.5$, the odds of non-survival were 6.39 times higher than in those with PDW $\leq 13.5$. Furthermore, the odds of mortality were 28.6 times more for the subjects with PLT $<0.11$ than for those with PLT $\geq 0.11$ (shown in Table 4).

\section{Discussion}

The frequently used platelet indices include PLT, MPV, PDW, and PCT. Although a few studies are evidencing the role of increased MPV in the etiopathogenesis of various disorders, lack of follow-ups has hindered standardization of MPV measurements. ${ }^{7}$ Standardized platelet indices values can aid quicker assessment of illness severity, follow-up, and reliable outcome prediction in critically ill patients, especially in resource-poor settings. ${ }^{8}$ Our study aimed to find the utility of platelet indices as prognostic markers in critically ill patients. Among the 106 patients studied, 82 patients survived, and 24 patients expired. The mortality in our study was $22.64 \%$, in agreement with the study conducted by Zhongheng Zhang MM. ${ }^{9}$

In the present study, diabetes mellitus (16.98\%) and hypertension $(16.98 \%)$ were the most common comorbid conditions. The findings are concordant with the study done by Bhattacharjee et al..$^{10}$ and Yang et al. ${ }^{11}$ Main reasons for abnormal PLT functions in diabetes mellitus (DM) are the immature, larger platelets as well as activated platelets due to the metabolic milieu in DM or due to vascular damage. All these factors have been proven to be interlinked. ${ }^{12}$ Some studies ${ }^{13,14}$ suggest that two possible mechanisms can control the increased levels of platelets during high blood pressure conditions. Firstly, pulmonary vascular endothelial dysfunction was linked to the path mechanisms of hypertension, which might lead to platelet activation and local thrombosis. Secondly,

Table 3. Mean values of platelet indices among survivors and non-survivors.

\begin{tabular}{lccl}
\hline Platelet indices & $\begin{array}{c}\text { Mean value - survivors } \\
(\mathbf{n = 8 2})\end{array}$ & $\begin{array}{c}\text { Mean value - non-survivors } \\
(\mathbf{n = 2 4 )}\end{array}$ & P-value \\
\hline Platelet count (cells/cumm) & $132,800 \pm 17,550$ & $77,500 \pm 10,570$ & $0.001^{*}$ \\
\hline PDW (\%) & $11.24 \pm 2.61$ & $13.6 \pm 1.63$ & $0.038^{*}$ \\
\hline MPV (fL) & $11.46 \pm 2.71$ & $15.2 \pm 1.46$ & $0.024^{*}$ \\
\hline Plateletcrit (\%) & $0.17 \pm 0.048$ & $0.11 \pm 0.031$ & $0.017^{*}$
\end{tabular}

PDW, platelet distribution width; MPV, mean platelet volume. *Significant at $\mathrm{P}<0.05$ threshold.

Table 4. Comparison of platelet indices with the outcome.

\begin{tabular}{llcccc}
\hline Platelet indices & & Non-survival & Survival & OR[95\% CI] & P-value \\
\hline Platelet count(in lakhs) & $<70,000$ & 2 & 0 & $18.33[0.85-395.70]$ & 0.0699 \\
& $\geq 70,000$ & 22 & 82 & & $0.0015^{*}$ \\
\hline PDW & $>13.5$ & 20 & 36 & $6.39[2.01-20.35]$ & 0.0909 \\
& $\leq 13.5$ & 4 & 46 & $2.70[0.85-8.58]$ & \\
\hline MPV & $>15$ & 6 & 9 & & $0.0005^{*}$ \\
& $\leq 15$ & 18 & 73 & $28.57[5.65-144.50]$ & \\
\hline Plateletcrit & $<0.11$ & 10 & 80 & & \\
& $\geq 0.11$ & 14 & & & \\
\hline
\end{tabular}

$\mathrm{OR}$, odds ratio; $\mathrm{CI}$, confidence interval; $\mathrm{PDW}$, platelet distribution width; $\mathrm{MPV}$, mean platelet volume. *Significant at $\mathrm{P}<0.05$ threshold. 
systemic inflammation and immune dysfunction in patients with high blood pressure might cause platelet activation. ${ }^{15}$

Here, the mean platelet count was $132,800 \pm 17,550$ cells/cumm among survivors and 77,500 $\pm 10,570$ cells/cumm among non-survivors. Significantly lower PLT count was noticed in non-survivors when compared to survivors. PLT count $<90,000$ cells/cumm had $94 \%$ specificity and $93 \%$ sensitivity in predicting mortality in critically ill patients. This was in concordance with the study done by Zhang et al., ${ }^{1}$ Bunyamin Burunsuzoglu et al., ${ }^{16}$ Seung Jun Choi et al., ${ }^{17}$ Patki et al., ${ }^{18}$ Koyama et al. ${ }^{19}$ also reckoned that diminished PC and $\alpha 2-P I$ activity was followed by a decline in platelet count among septic intensive care unit patients.

The mean PDW in our study was found to be $11.24 \pm 2.61 \%$ among survivors and $13.6 \pm 1.63 \%$ among non-survivors, and the difference was statistically significant. In critically ill patients' mortality prediction, a PDW of $>13.5 \%$ had $90 \%$ specificity and $96 \%$ sensitivity. Similar results were observed in other studies by Guclu et al., ${ }^{20}$ Sheng Zhang et al., ${ }^{1}$ Fogagnolo et al. ${ }^{21}$ found that the PDW values in the predic-

ROC curve for platelet count:

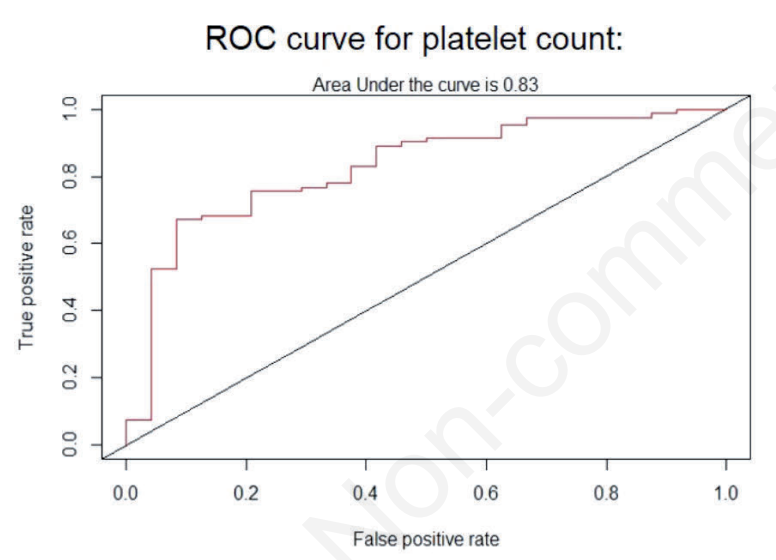

ROC curve for MPV:

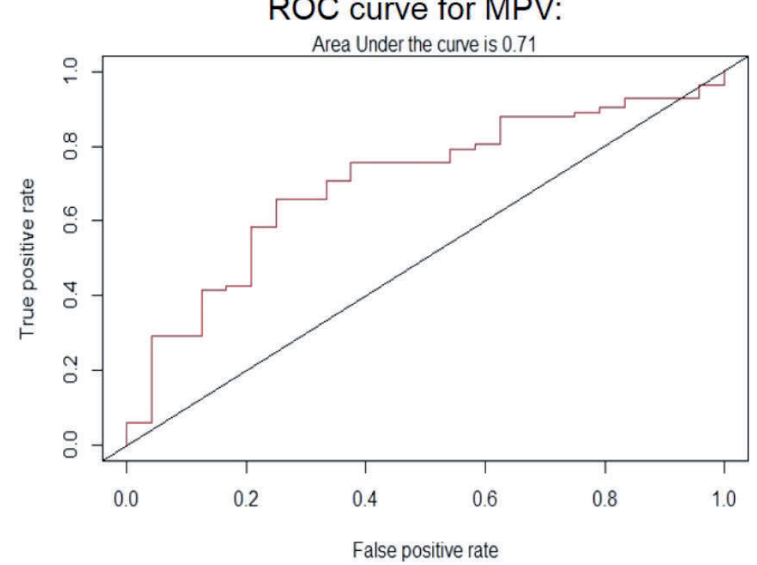

tion of 90-day mortality were significant in septic patients but not in non-septic patients $(\mathrm{P}<0.001)$.

The mean plateletcrit was $0.17 \pm 0.048 \%$ among survivors and $0.11 \pm 0.031 \%$ among non-survivors, with a significant difference between the mean values of plateletcrit between survivors and non-survivors $(\mathrm{P}=0.017)$. A plateletcrit of $<0.11 \%$ had $91 \%$ sensitivity and a $95 \%$ specificity in mortality prediction. The odds ratios for mortality prediction concluded that PCT and PDW were two significant factors affecting the subjects' survival status. For patients with PDW $<13.5$, the odds of non-survival were 6.39 times higher than for those with PDW $\leq 13.5$. Also, the odds of mortality were 28.6 times higher for the subjects with plateletcrit $<0.11$ than for those with plateletcrit $\geq 0.11$. Our study results were consistent with various other studies such as by Zhang et al. ${ }^{1}$ and Golwala et al. ${ }^{22}$

Average MPV values noted among survivors and non-survivors showed statistically significant variations between the two groups. This is reinforced by the findings of Tajarernmuang et al., ${ }^{23}$ who extrapolated that after three days from patients admission, the MPV was significantly higher among non-survivors.
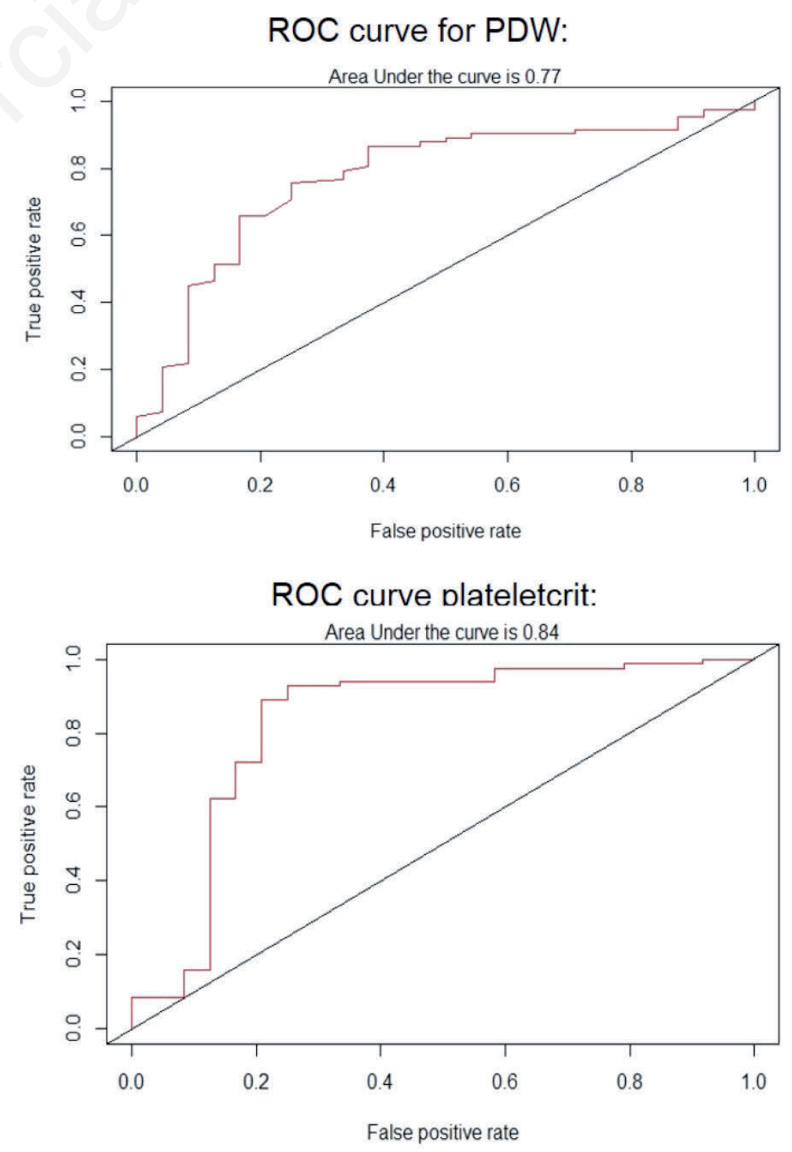

Figure 2. Receiver operating characteristic (ROC) curves for platelets count, platelet distribution width (PDW), mean platelet volume (MPV), and plateletcrit. 
In our study, PLT count, PDW, MPV, and PCT possessed large areas under receiver operating characteristic (ROC) of $0.83,0.77,0.71$, and 0.84 , respectively. Among all four indices, PCT ranked supreme for predictive accuracy. Thus, PCT was inferred to be the optimal predictive indicator. This contrasts with the study done by Zhang et al. ${ }^{1}$ where MPV was the optimal predictive indicator studied. Liberski et al., ${ }^{24}$ however, note that PLT/MPV are not reliable predictors of sepsis. In consonance with our findings, Samuel et al. demonstrated a significant influence of PLT indices, including PLT, PDW, PCT, and MPV adjusted to DM by a univariate regression analysis. Samuel et al. also observed that patients having low PCT, PLT values along with high levels of MPV and PDW were at a higher risk of severe illness, worsening prognosis, and mortality. ${ }^{25}$

This study has certain limitations, such as only PLT indices were analyzed. Other disseminated intravascular coagulation parameters (prothrombin time, activated partial thromboplastin time, or D-dimer) were not recorded and analyzed in this study, which may be a major confounding factor. Another limitation is that details regarding the cause of death among non-survivors were not noted. Hence, future studies can be designed by eliminating the limitations mentioned above.

Although platelet count is a part of several clinically used scoring systems such as Sequential Organ Failure Assessment (SOFA), APACHE II, and APACHE III, platelet indices such as MPV (routinely included in hematology reports) are often overlooked parameters for clinical interpretation. In this study, we show that these platelet indices, when deranged, can also be useful in the prognostication of critically ill patients and must be taken into account in routine clinical practice. However, we suggest that further studies in larger groups and multiple settings are essential to investigate the impact of incorporating these platelet indices into the currently used scoring systems to increase their accuracy of prognosticating septic and non-septic survivors and non-survivors.

\section{Conclusions}

The severity of illness, as well as the clinical outcomes, can be accurately predicted by platelet indices. Abnormally low PLT count, high PDW, and high MPV are associated with severe illness. In addition, reduced PLT count and PCT or increased MPV and PDW put the patients at high risk of death compared to patients with normal PLT indices. Platelet indices can be utilized as simple, inexpensive tools for prognostication in critically ill patients and must be validated by extensive multi-centric studies for incorporating them into standard clinical practice.

\section{References}

1. Zhang S, Cui YL, Diao MY, et al. Use of platelet indices for determining illness severity and predicting prognosis in critically ill patients.Chin Med J 2015;128:2012-8.

2. Budak YU, Polat M, Huysal K. The use of platelet indices, plateletcrit, mean platelet volume and platelet distribution width in emergency non-traumatic abdominal surgery: a systematic review. Biochem Med (Zagreb) 2016;26:178-93.

3. Kantharaj A. Role of red cell and platelet indices as a predictive tool for transfusions in dengue. Glob J Transfus Med 2018;3:103-8.

4. Catal F, Tayman C, Tonbul A, et al. Mean platelet volume may simply predict the severity of sepsis in preterm infants. Clin Lab 2014;60:1193-200.

5. Yoo H, Ku SK, Kim SW, Bae JS. Early diagnosis of sepsis using serum hemoglobin subunit Beta. Inflammation 2015;38:394-9.

6. Simani L, Oroei M, Sadeghi H, et al. Platelet indices for predicting patient outcomes in post-neurosurgical meningitis. Arch Neurosci 2019;6:e82911.

7. Bayir H, Yildiz I. The importance of platelet markers on hospital mortality in intensive care unit patients. J Crit Care 2015;30:433.

8. Zhang Z. Platelet indices in the prognostication of critically ill patients: another piece of the puzzle. J Crit Care 2015;30:434-5.

9. Zhang Z, Xu X, Ni H, Deng H. Platelet indices are novel predictors of hospital mortality in intensive care unit patients. J Crit Care 2014;29:885.e1-6.

10. Bhattacharjee, Datta A, Debbarma RK, Das SK. Platelet indices in diabetics and influence of glycemic control a hospital based study in North-East India. Int J Med Res Rev 2016;4:2186-2192.

11. Yang K, Tao L, Mahara G, et al. An association of platelet indices with blood pressure in Beijing adults: Applying quadratic inference function for a longitudinal study. Medicine (Baltimore) 2016;95:e4964.

12. Yngen M. Platelet hyperactivity in diabetes mellitus. Eur Cardio 2005;1:1-6.

13. Uçar H, Gür M, Gözükara MY, et al. Relationship between mean platelet volume and morning blood pressure surge in newly diagnosed hypertensive patients. Anatol J Cardiol 2015;15:107-12.

14. Pusuroglu H, Cakmak HA, Erturk M, et al. Assessment of the relation between mean platelet volume, non-dipping blood pressure pattern, and left ventricular mass index in sustained hypertension. Med Sci Monit 2014;20:2020-6.

15. Zheng YG, Yang T, Xiong CM, et al. Platelet distribution width and mean platelet volume in idiopathic pulmonary arterial hypertension. Heart Lung Circ 2015;24:566-72.

16. Burunsuzoglu B, Salturk C, Karakurt Z, et al. A risk factor of mortality for patients with sepsis in the Intensive Care Unit. Turk Thorac J 2015;17:7-14.

17. Choi SJ, Ha E, Jhang WK, Jong Park S. Platelet indices as predictive markers of prognosis in pediatric septic shock patients. Iran J Pediatr 2017;27:e7212.

18. Patki VK, Birru FV, Patki VV. Dsecline in platelet count as a prognostic marker in critically ill children. J Pediatr Crit Care 2014;1:13-21. 
19. Koyama K, Madoiwa S, Tanaka S, et al. Evaluation of hemostatic biomarker abnormalities that precede platelet count decline in critically ill patients with sepsis. J Crit Care 2013;28:556-63.

20. Guclu E, Durmaz Y, Karabay O. Effect of severe sepsis on platelet count and their indices. Afr Health Sci 2013;13:333-8.

21. Fogagnolo A, Taccone FS, Benetto G, et al. Platelet morphological indices on ICU admission predict mortality in septic but not in non-septic patients. Minerva Anestesiol 2020 Sep 22. [Epub ahead of print].

22. Golwala ZM, Shah H, Gupta N, et al. Mean platelet volume (MPV), platelet distribution width (PDW), platelet count and plateletcrit (PCT) as predictors of in hospital paediatric mortality: a case - control study. Afr Health Sci 2016;16:356-62.

23. Tajarernmuang P, Phrommintikul A, Limsukon A, et al. The role of mean platelet volume as a predictor of mortality in critically ill patients: a systematic review and meta-analysis. Crit Care Res Pract 2016;2016:4370834.

24. Liberski PS, Szewczyk M, Krzych ŁJ. Haemogram-derived indices for screening and prognostication in critically ill septic shock patients: a case-control study. Diagnostics (Basel) 2020;10:638.

25. Samuel D, Bhat AN, Prabhu VM. Platelet indices as predictive markers of prognosis in critically ill patients: a prospective study. Indian J Crit Care Med 2020;24:817-22. 